\title{
PERFIL ECOLÓGICO DE CUATRO RODALES DE CAMU CAMU ÁRBOL Myrciaria floribunda (H. West. ex Willd) O. Berg. EN UCAYALI
}

\section{ECOLOGICAL PROFILE OF FOUR RODALS OF ARBOREAL CAMU CAMU Myrciaria floribunda (H. West. ex Willd) O. Berg IN UCAYALI}

\author{
Antonio López ${ }^{1}$, Enrique Bicerra ${ }^{2}$ y Edgar Díaz ${ }^{3}$
}

\begin{abstract}
Resumen
El presente estudio describe el perfil ecológico de camu camu árbol Myrciaria floribunda $\mathrm{H}$. West ex Wild O. Berg. en base a la caracterización de cuatro rodales ubicados en la llanura aluvial periódicamente inundable de la cuenca del río Ucayali, a una altitud promedio de $155 \mathrm{msnm}$. La composición florística de los cuatro rodales es homogénea, con cocientes de mezcla promedio de 1/10 y no superan los $30 \mathrm{~m}^{2}$ de área basal total, por lo que se consideran como sitios de baja fertilidad natural y en etapa de sucesión temprana. De acuerdo al IVIA, en los tres rodales (Caco Macaya, Agua Negra y Sábalo), Myrciaria floribunda se presenta entre las seis especies de mayor importancia ecológica, presentando una distribución natural agrupado, con distribución horizontal no continua. En cuanto a la regeneración natural de Myrciaria floribunda, aún cuando no existe una escala de referencia para la especie, se considera que en promedio es alta con 3208 individuos por hectárea.
\end{abstract}

Palabras claves: calidad de sitio, camu camu árbol, diversidad florística, suelos aluviales

\begin{abstract}
The present study describes the ecological profile of the arboreal camu camu Myrciaria floribunda $\mathrm{H}$. West ex-Wild O. Berg. taking into account the characterization of four rodals located in the periodically flooded alluvial terraces of the Ucayali River Basin, at an average altitude of 155 m.a.s.l. The floristic composition of four rodals is homogeneous, with average mixing quotients of $1 / 10$ that do not overcome $30 \mathrm{~m}^{2}$ of the total basal area. Due to this, they are considered as low natural fertility places and in early succession stages. In accordance to IVIA, in three rodals (Caco Macaya, Agua Negra and Sábalo), Myrciaria floribunda is among the six species of major ecological importance, presenting a grouped natural distribution with a non continuous horizontal distribution. Regarding the natural regeneration of Myrciaria floribunda, even though a scale of reference for the species does not exist, an average of 3208 individuals per hectare is considered high.

Words keys: quality of place, arboreal camu camu, floristic diversity, alluvial soils
\end{abstract}

\section{Introducción}

El conocimiento de los bosques amazónicos y sus alternativas de producción han sido durante muchos años, producto del esfuerzo de las investigaciones que han derivado en propuestas de desarrollo con enfoques conservacionistas y expansionistas.

Así, Lamprecht (1990), menciona que el estudio de su estructura es importante en las investigaciones silviculturales porque permite efectuar deducciones importantes del origen, dinamismo y tendencias del futuro desarrollo de las comunidades forestales; ofrecen datos sobre las condiciones de hábitat y su influencia formativa de los árboles del trópico y son bases importantes para poder delinear las técnicas silviculturales a aplicar.

Según Finol (1971), la constancia de la distribución diamétrica en un bosque húmedo tropical no disturbado semeja a una curva exponencial y encierra en sí un significado fito sociológico en el desarrollo del bosque hacia el clímax. Sin embargo, a nivel de especie, el análisis se hace difícil porque ciertas especies de importancia comercial que usualmente ocupan una posición superior en el dosel arbóreo salen del esquema típico y tienen un proceso de regeneración y crecimiento que hace variar esta curva.

Por su parte, Braun-Blanquet (1979) usa un Índice de Valor de Importancia Ampliado (IVIA) para cada especie al analizar bosques templados. Este Índice ha sido aplicado por varios investigadores del trópico amazónico como Sabogal (1980); y Salcedo (1986) quienes sostienen que bosques pueden ser descritos cuantitativamente mediante el componente florístico y cualitativamente con relación a la estratificación vertical y horizontal de los árboles.

Respecto al medio en que se desarrollan, Hubell \& Foster (1990) encontraron que algunas especies forestales presentan una estrecha relación con las características fisiográficas, y Malleux (1982) considera que los bosques de terrazas bajas, tienen áreas planas con drenaje regular a malo, altura relativa sobre el nivel del río de 5 a 10 metros, vigor de la 
vegetación de moderado a bueno y terreno relativamente plano con algunas depresiones.

Por otro lado, Kalliola et al. (1993), mencionan, que el principal criterio usado en la caracterización y clasificación del agua en el campo es el color. Los llamados ríos de "agua blanca” presentan una gran cantidad de sólidos en suspensión, mientras que los ríos de "agua negra” son ricos en sustancias húmicas.

Camu camu árbol se constituye en una fuente complementaria a la especie arbustiva y (Myrciaria dubia HBK) cultivada en las regiones de Loreto y Ucayali en razón a su alto contenido de vitamina C (1526 mg/100gr de pulpa) siendo atractiva para el mercado internacional que requiere ingentes cantidades de pulpa congelada de camu camu, según refieren varios investigadores como Imán (2000) y Pinedo (2001).

Sobre el particular, López (2005) menciona que la producción de fruta de esta especie se realiza anualmente entre los meses de diciembre a marzo, cuando la especie arbustiva está iniciando su fase de floraron, pero por la poca producción solo satisface el mercado local.

Sin embargo, trabajos de investigación sobre la especie se reportan en escasa literatura. Así, Mendoza et al. (1989) manifiestan que en forma natural se encuentra en las inmediaciones de los ríos Ucayali, Napo, Nanay, y en las cochas Sahua y Supay 2 asi como cerca de los caseríos Cashibococha, Quebrada Tahuantinsuyo, Río Manantay y Yarinacocha.

Por su parte, Villachica (1996) sostiene que Myrciaria sp. es un árbol de hasta $15 \mathrm{~m}$. de altura, con ramas glabras, hojas oblondo - elípticas de 6.0 a 12.0 cm de largo y de 2.5 a $3.5 \mathrm{~cm}$ de ancho, ápice acuminado y base cuneada a obtusa, glabras con un nervio medio impreso en el haz y con numerosos nervios secundarios subparalelos y más o menos perpendiculares al nervio medio. Las inflorescencias son axilares, en címulas de 2 a 3 flores, glabras y blancas. Los frutos son de 2 a $3 \mathrm{~cm}$ de diámetro, rojizos a negros cuando maduros.

Consecuentemente, el presente estudio tuvo por objetivo describir las condiciones fisiográficas, edáficas, hídricas y florísticas de cuatro rodales donde camu camu arbol forma parte de su estructura.

\section{Materiales y métodos}

El área de estudio se ubica en la región Ucayali, provincia de Coronel Portillo y distritos de Iparia y Callería y abarca una superficie de 103.2 ha de las planicies de inundación de los ríos Caco Macaya, Agua Negra, Sábalo y Agua Blanca. Cada rodal fue designado con el nombre de los ríos adyacentes (Figura 1').

La temperatura promedio fue de $31.1{ }^{\circ} \mathrm{C}$, precipitación anual $1586 \mathrm{~mm}$, humedad relativa $84.2 \%$ y evaporación promedio 57.5 mm (UNU, 2002).

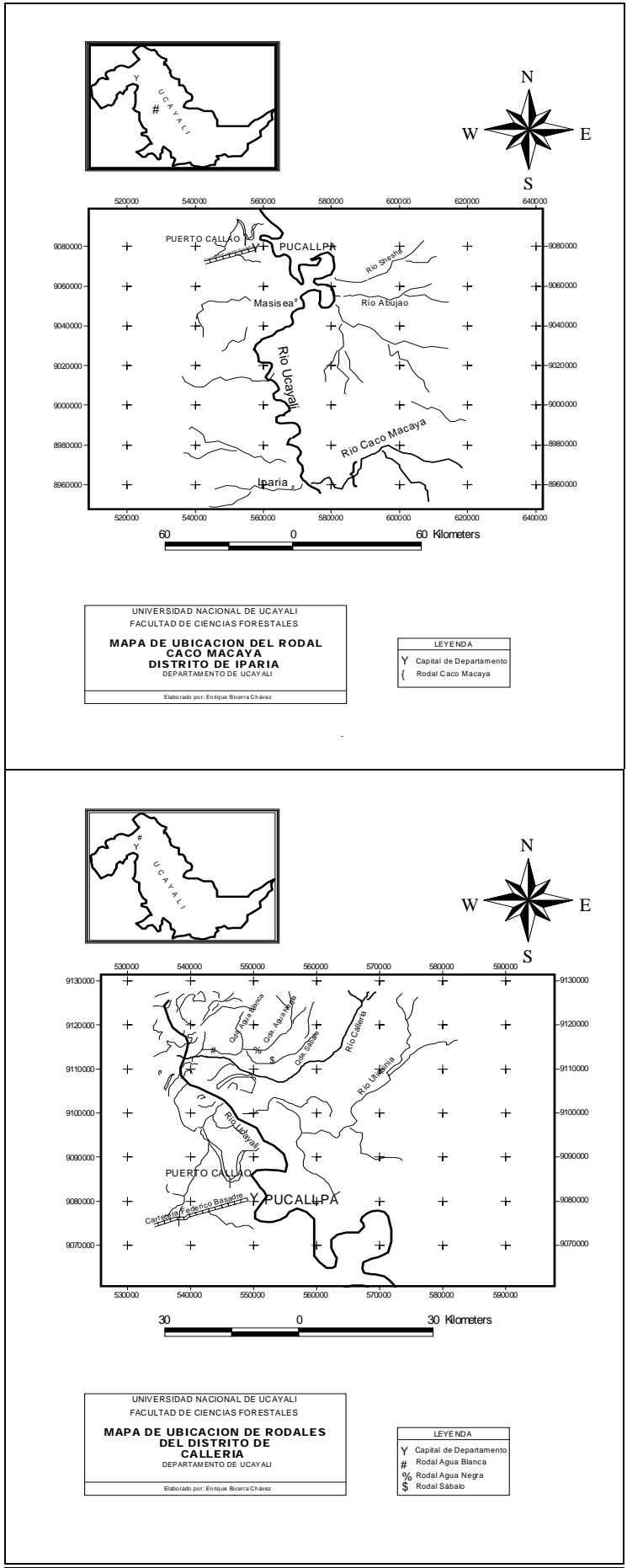

Figura 1'. Mapa de ubicación de los rodales de Camu camu, Myrciaria floribunda (H. West. ex Willd) O. Berg. en Ucayali.

El método empleado fue descriptivo y analítico. Por cada rodal, se levantaron dos parcelas de evaluación de 20 × 250 m ó 40 x 130 m para fustales, 10 x 10 m, para latizales y 2 x 2 m para brinzales, con 
el objeto de medir las variables de vegetación, fisiografía y suelo.

La determinación taxonómica de las especies inventariadas en estas parcelas fue realizada mediante la colecta de muestras botánicas por cada rodal, las cuales una vez preparadas, fueron llevadas al Instituto Veterinario de Investigaciones Tropicales y de Altura (IVITA) para su identificación en base al Herbario de Especies Amazónicas, con que cuenta dicha entidad.

El estudio de la vegetación considera los parámetros que determinan la estructura horizontal y vertical de las especies, incluyendo en estos últimos el componente de la regeneración natural, según categorías de tamaño (Sabogal, 1980).

Con relación a la estructura horizontal, se evaluó la abundancia absoluta (Ab) y relativa (Ab\%), la dominancia absoluta y relativa y la frecuencia absoluta y relativa, las cuales se clasificaron según los grupos de frecuencia utilizados por Lamprecht (1990).

Con relación a la estructura vertical se evaluaron la posición sociológica absoluta y relativa.

El análisis de la regeneración natural se hizo en base al número de individuos menores a $10 \mathrm{~cm}$ de diámetro a la altura del pecho (dap) de las especies de árboles y palmeras. Se consideraron tres categorías de tamaño (c.t.) para cada especie (Tabla 1).

Tabla 1. Características de las parcelas de regeneración natural.

\begin{tabular}{cccc}
\hline Nro & $\begin{array}{c}\text { Categoría. de } \\
\text { tamaño (c.t.) }\end{array}$ & $\begin{array}{c}\text { Tamaño de } \\
\text { parcela (m) }\end{array}$ & Intervalo de evaluación \\
\hline 01 & Brinzales & $2 \times 2$ & 0.1 a 1.49 m de altura \\
02 & $\begin{array}{c}\text { Latizal Bajo } \\
\text { Latizal alto }\end{array}$ & $10 \times 10$ & $1.5 \mathrm{~m}$ a 2.99 m de altura \\
03 & & $3.0 \mathrm{~m}$ de altura a $9.9 \mathrm{~cm}$ de dap \\
\hline
\end{tabular}

Fuente: Sabogal (1980)

Para cada especie se calculó las siguientes variables: abundancia absoluta (Ab.) y relativa (Ab.\%); frecuencia absoluta (Fr.) y relativa (Fr \%) y categoría de tamaño absoluto (C.t.) y relativa (C.t.\%)

Para los dos primeros el cálculo fue similar al del análisis de la estructura horizontal.

La categoría de tamaño tuvo el mismo criterio fitosociológico que para el cálculo de la posición sociológica.

Finalmente el parámetro Regeneración natural R.n.\% de cada especie se obtuvo por medio de la siguiente fórmula:

R.n.\% = Ab.\%R.n. + Fr.\%R.n. + C.t.\% R.n./ 3

Por otro lado, el índice de valor de importancia ampliado (IVIA) fue obtenido al sumar los valores relativos de los parámetros de la estructura horizontal para cada especie de acuerdo a la siguiente expresión:

I.V.I.A.= Ab. $\%+$ Fr. $\%$ + D. $\%+$ P.s. $\%+$ R.n. $\%$
La suma total de estos porcentajes para todas las especies (árboles + palmeras) da un valor de 500 . Para el caso de la asociación con especies forestales se consideró las especies forestales ligadas a la presencia del camu camu arbóreo, mediante la determinación del Índice de valor de importancia (ÍVI).

Para clasificar la distribución de las especies se utilizó el método de patrones de agrupamiento empleado por Equihua (1994):

- Distribución al azar: cuando la distancia promedio entre árboles de camu camu es igual a la varianza de las distancias

- Agrupados o en grupos: Cuando la distancia promedio entre árboles de camu camu es menor que la varianza de las distancias

- Distribución regular: Cuando la distancia promedio entre árboles de camu camu es mayor que la varianza de las distancias.

La clasificación de agua, se basa en la usada por Sioli citada por Kalliola (1993), la cual usa el color como criterio de clasificación basado en una estrecha relación con las propiedades químicas. Las categorías consideradas en este estudio son : agua blanca: color semejante al café con leche,. agua negra: color similar al café negro y agua clara: transparentes, usualmente de color verdoso

La evaluación del suelo se hizo en dos etapas: primero se realizó la apertura de un perfil modal en cada rodal, en áreas representativas de las parcelas, posteriormente se hizo una descripción detallada de los perfiles (horizontes) aperturados usando formato o tarjetas de descripción que utiliza la UNA La Molina y el INIA - Yurimaguas.

El análisis físico y químico de las muestras recolectadas, se realizaron en el laboratorio de suelos y tejidos vegetales del INIA - Pucallpa.

Para la clasificación de suelos, se usó el Sistema de Soil Taxonomy (USDA NRCS, 1998) y sus modificaciones, ya que sus criterios cuantitativos hacen que éste sea el sistema de clasificación más apropiado.

\section{Resultados y discusión}

Análisis de la estructura horizontal

Caco Macaya es el rodal de mayor densidad poblacional, con 472 individuos por ha, mientras que el rodal que presenta menor densidad es Agua Blanca, con 281 individuos por ha), probablemente a consecuencia de las intervenciones realizadas por los pobladores ribereños. El rodal de Myrciaria floribunda con mayor abundancia, es Agua Negra, seguido por los rodales Sábalo, Caco Macaya y Agua Blanca (Tabla 2).

Respecto a frecuencia, Caco Macaya es el único rodal que presenta las clases frecuenciales III y IV, las cuales están representadas por las especies caimitillo (Abuta grandifolia) y tamalillo respectivamente, siendo las especies que presentan mejor distribución 
horizontal en la superficie del rodal; a excepción de estas dos especies, todas las demás especies (incluido Myrciaria floribunda), en los rodales estudiados, presentan frecuencia tipo I y II, clasificándoles como especies de distribución horizontal no continua, Lamprecht (1990).

Tabla 2. Superficie y características dasométricas de los cuatro rodales.

\begin{tabular}{ccccccccccc} 
Rodal & $\begin{array}{c}\text { Superf. } \\
\text { (ha) }\end{array}$ & Sp/ha & C.M. ${ }^{1}$ & $\begin{array}{c}\text { Abundancia } \\
\text { Ind./ha }\end{array}$ & $\begin{array}{c}\text { Dominancia } \\
\mathrm{m}^{2} / \text { ha }\end{array}$ & $\begin{array}{c}\text { Frecuencia } \\
\text { Tipo }^{3}\end{array}$ \\
\cline { 5 - 10 } & & & & & Rodal & M.f. ${ }^{2}$ & Rodal & M.f. $^{2}$ & Rodal & M.f. $^{2}$ \\
\hline Caco Macaya & 60 & 41 & $1 / 12$ & 472 & 24 & 21 & 1.32 & I - IV & I \\
Agua Negra & 16.8 & 39 & $1 / 8$ & 322 & 32 & 16 & 1.13 & I,II & II \\
Sábalo & 14.4 & 26 & $1 / 12$ & 312 & 31 & 15.7 & 1.32 & I,II & II \\
Agua Blanca & 12 & 28 & $1 / 10$ & 281 & 12 & 28.9 & 0.51 & I,II & I \\
\hline
\end{tabular}

${ }^{1}$ C.M. (Cociente de mezcla)

2 M.f. (Myrciaria floribunda)

${ }^{3}$ Lamprecht 1990

Con relación a dominancia, Agua Blanca, es el rodal que presenta mayor área basal absoluta seguido por los rodales Caco Macaya, Agua Negra y Sábalo, sin embargo estos rodales están por debajo del valor promedio (38 $\mathrm{m}^{2} / \mathrm{ha}$ ), estimado por Hoheisel, 1976, (citado por Kalliola et al., 1993) para bosques tropicales indicándonos que existe abundante presencia de especies de mediana categoría diamétrica y/o que el rodal aún se encuentra en etapa sucesional.

En los cuatro rodales, aproximadamente el $75 \%$ del área basal total, está representado aproximadamente por el $15 \%$ de las especies, confirmando lo referido por Lamprecht (1990), quien menciona que menos del $15 \%$ de las especies arbóreas, en bosques tropicales, comprenden un 50 a $70 \%$ del área basal total.

La distribución diamétrica en los cuatro rodales es regular (Figuras 1A, 1B, 1C y1D), semejante a una curva exponencial, el cual manifiesta la característica de un bosque húmedo tropical no disturbado, y de un significado fitosociológioco de desarrollo del bosque hacia el climax.

La distribución diamétrica regular, implica que las categorías inferiores incluyen el suficiente número de individuos que se requiere para reemplazar a los árboles grandes que mueren en forma natural y/o por intervención, coincidiendo con los estudios realizados por Lamprecht (1990), en bosques tropicales. Sin embargo, el rodal de Agua blanca presenta irregularidades en su distribución diamétrica, probablemente a consecuencia de intervenciones, debido a que este rodal se encuentra cercano a comunidades y caseríos.

Por su parte, camu camu arbóreo presenta distribución diamétrica normal en todos los rodales, sin embargo en relación con el desarrollo de los fustales jóvenes en los rodales Caco Macaya, Agua
Negra y Sábalo (figuras 2A, 2B, 2C y 2D) se observa un "cuello de botella", lo que probablemente se deba a la interrupción en el proceso de sustitución natural de árboles adultos por jóvenes. El análisis de la distribución diamétrica de Myrciaria floribunda, coincide lo sustentado por Finol (1974), quien menciona que el análisis de la distribución diamétrica a nivel específica se hace difícil ya que ciertas especies, como Myrciaria floribunda, con individuos que usualmente ocupan una posición superior en el dosel arbóreo, salen del esquema típico y tienen un proceso de regeneración y crecimiento que hace variar la curva, lo cual lleva a pensar en el virtual peligro de desaparición de la especie, siendo este un proceso natural de la sucesión

forestal.

En forma natural, el patrón de distribución de los individuos de Myrciaria floribunda en los cuatro rodales es Agrupado, Equihua (1994), registrándose mayor número de individuos en zonas depresionadas (cóncavas) y mal drenadas, a consecuencia posiblemente, de los requerimientos muy específicos de factores fisiográficos y/o edáficos de la especie, confirmando lo sustentado por Bruning (1970), quien asegura que dentro de una zona de vida bio climáticamente uniforme, las propiedades físicas y químicas del suelo son los principales modificadores de la estructura intra o Inter específica del bosque, en el cual probablemente el factor drenaje esté jugando un rol importante.

Análisis de la estructura vertical

En términos generales, los rodales presentan el mayor número de individuos en el estrato inferior (más del $50 \%$ ), lo cual confirmaría el análisis de distribución diamétrica, que los rodales presentan los suficientes individuos jóvenes para el reemplazo de árboles maduros, a excepción del rodal Agua Blanca, que aparentemente presenta intervenciones humanas, por tal motivo exista irregularidad en la presentación de su estratificación, el cual presenta el mayor porcentaje de individuos en estrato medio. Tabla 3.

Myrciaria floribunda presenta distribución vertical continua (presente en los tres estratos). En tres rodales presenta más del $50 \%$ de su población dentro la estratificación inferior (Tabla 4), excluyendo el rodal Caco Macaya, el cual concentra el $71 \%$ de su población en el estrato medio, por tal motivo es necesario cuestionar su preservación, coincidiendo con el análisis de distribución diamétrica, que la especie presenta un virtual peligro de desaparición. 
Tabla 3. Estratificación de la población arbórea de los cuatro rodales.

\begin{tabular}{|c|c|c|c|}
\hline \multirow[t]{2}{*}{ Rodales } & \multicolumn{3}{|c|}{ Estratos } \\
\hline & Inferior(\%) & Medio(\%) & Superior(\%) \\
\hline Caco Macaya & 58 & 38 & 4 \\
\hline Agua Negra & 59 & 34 & 7 \\
\hline Sábalo & 57 & 39 & 4 \\
\hline Agua Blanca & 44 & 49 & 7 \\
\hline
\end{tabular}

Tabla 4. Estratificación de Myrciaria floribunda en los cuatro rodales.

\begin{tabular}{|c|c|c|c|}
\hline \multirow[t]{2}{*}{ Rodales } & \multicolumn{3}{|c|}{ Estratos } \\
\hline & Inferior(\%) & Medio(\%) & Superior(\%) \\
\hline Caco Macaya & 21 & 71 & 8 \\
\hline Agua Negra & 58 & 39 & 3 \\
\hline Sábalo & 50 & 50 & 0 \\
\hline Agua Blanca & 58 & 42 & 0 \\
\hline
\end{tabular}

Regeneración Natural

En promedio, el número de individuos en la etapa regenerativa de los rodales naturales fue de 19004 individuos por ha, de los cuales el 17\% (3208), está representado por la especie Myrciaria floribunda. El mayor número de individuos de regeneración natural de Myrciaria floribunda se encuentra en la categoría de brinzales representando el $97 \%$ de la población total; solo el $2 \%$ relativo a los brinzales son representados por la categoría latizales bajos, de las cuales el 29\% son representados por los latizales altos (Tabla 5) En tal sentido existe un "cuello de botella”, en el desarrollo de brinzales a latizales de la especie en estudio, a causa probablemente de las inundaciones que sufren los terrenos de los rodales y a las exigencias progresivas de luz que exigen los individuos.

Tabla 5. Regeneración natural de los cuatro rodales y de Myrciaria floribunda.

\begin{tabular}{|c|c|c|c|c|c|}
\hline \multirow[t]{3}{*}{ Rodales } & \multicolumn{4}{|c|}{ Regeneración Natural } & \multirow[t]{3}{*}{ TOTAL $^{1}$} \\
\hline & \multirow{2}{*}{ Rodal } & \multicolumn{3}{|c|}{ Myrciaria floribunda } & \\
\hline & & Brinzales & Lat.bajo & Lat.Alto & \\
\hline Caco Macaya & 18493 & 1709 & 203 & 17 & 1929 \\
\hline Agua Negra & 14923 & 5523 & 38 & 28 & 5589 \\
\hline Sábalo & 27738 & 4618 & 12 & 29 & 4659 \\
\hline Agua Blanca & 14860 & 631 & 18 & 6 & 655 \\
\hline TOTAL & 76014 & 12481 & 271 & 80 & 12832 \\
\hline PROMEDIO & 19004 & 3120 & 68 (2\%) & $\begin{array}{c}20 \\
(29 \%)\end{array}$ & 3208 \\
\hline
\end{tabular}

${ }^{1}$ Total de individuos de regeneración natural de Myrciaria floribunda
Índice de Valor de Importancia Ampliado (IVIA)

De acuerdo a los índices de valor de importancia ampliado (IVIA), Myrciaria floribunda, se encuentra dentro de especies de mayor importancia ecológica en los rodales Caco Macaya, Agua Negra y Sábalo, con el sexto, segundo y tercer lugar, respectivamente, mientras que en el rodal Agua Blanca, no se encuentra entre las primeras especies de importancia ecológica, considerándosela, en este rodal, como especie "acompañante", según refiere Lamprecht (1990). En tal sentido se considera a los rodales Agua Negra, Sábalo y Caco Macaya, con las características más idóneas para el desarrollo de la especie (Tabla 6, en Apéndice).

Caracterización hídrica.

Respecto a la caracterización hídrica, las quebradas Caco Macaya, Agua Negra y Sábalo son de "agua negra", debido, según Kalliola et al. (1993) a su alto contenido de sustancias húmicas, como efecto de la acumulación y descomposición de materia orgánica en los suelos de los rodales. La quebrada Agua Blanca, tiene por su parte "agua blanca", como consecuencia, según el mismo autor, a la gran cantidad de sólidos suspendidos (Tabla 7).

\section{Caracterización edáfica}

Los suelos de los cuatro rodales pertenecen al Orden Entisols, Sub orden Fluvents, Gran grupo Udifluvents y Sub grupo Oxiaquic udifluvents.

Por las descripciones de sitio, las características morfológicas y los datos de los análisis físicos y químicos, se deduce que los suelos no presentan desarrollo pedogenético considerable. El suborden implica que los suelos se han desarrollado a partir de deposiciones aluviales relativamente recientes, en donde las inundaciones frecuentes no permiten el desarrollo de horizontes, de manera que lo que se encuentran en esas zonas son secuencias de capas de sedimentos, Los suelos de los cuatro rodales presentan predominio de fracciones finas y son de textura arcillosa, con ligeras variaciones texturales (Tabla 8).

Tabla 7. Clasificación, nivel y periodo de inundación de los rodales de Myrciaria floribunda.

\begin{tabular}{|c|c|c|c|}
\hline Rodal & Clasificación & $\begin{array}{l}\text { Nivel de } \\
\text { Inundación }\end{array}$ & $\begin{array}{l}\text { Periodo de } \\
\text { Inundación }\end{array}$ \\
\hline \multicolumn{4}{|l|}{ Caco } \\
\hline Macaya & agua negra & $8 \mathrm{~m}$ & Diciembre a Marzo \\
\hline Agua Negra & agua negra & $10 \mathrm{~m}$ & Noviembre a Abril \\
\hline Sábalo & agua negra & $10 \mathrm{~m}$ & Noviembre a Abril \\
\hline Agua Blanca & agua blanca & $9 \mathrm{~m}$ & Diciembre a Marzo \\
\hline
\end{tabular}

\section{${ }^{1}$ Kalliola (1993)}

El color de los suelos es una evidencia clara de la periodicidad de inundación. Los rodales Agua Negra y Sábalo presentan suelos de color gris, siendo este color característico de suelos reducidos (saturados de agua). 
Por otro lado, Caco Macaya y Agua Blanca presentan suelos de color pardo, característicos de suelos oxidados (oxigenados). Sobre la base de lo descrito se puede deducir que los suelos de los rodales Agua Negra y Sábalo soportan periodos más largos de inundación en comparación a los rodales Caco Macaya y Agua Blanca.

Tabla 8. Características físicas y químicas de los suelos de los cuatro rodales.

\begin{tabular}{|c|c|c|c|c|}
\hline \multirow[t]{2}{*}{ Característica } & \multicolumn{4}{|c|}{ Rodal } \\
\hline & $\begin{array}{c}\text { Сaco } \\
\text { Масауа }\end{array}$ & $\begin{array}{l}\text { Agua } \\
\text { Negra }\end{array}$ & Sábalo & $\begin{array}{c}\text { Agua } \\
\text { Blanca }\end{array}$ \\
\hline Textura & $\begin{array}{c}\text { Arcillo } \\
\text { Limoso }\end{array}$ & Arcilloso & Arcilloso & $\begin{array}{l}\text { Franco } \\
\text { arcillo- } \\
\text { limoso }\end{array}$ \\
\hline Color $^{1}$ & Pardo & $\begin{array}{c}\text { gris } \\
\text { oscuro }\end{array}$ & $\begin{array}{c}\text { gris } \\
\text { oscuro }\end{array}$ & $\begin{array}{l}\text { pardo } \\
\text { oscuro }\end{array}$ \\
\hline $\mathrm{pH}$ & 3.9 & 4.4 & 4.2 & 4.3 \\
\hline Mat. Org. (\%) & 3.7 & 5.9 & 5.8 & 1.1 \\
\hline C.I.C. ${ }^{2}$ & 16 & 21 & 20 & 10 \\
\hline
\end{tabular}

\footnotetext{
${ }^{1}$ Munsell Color

${ }^{2}$ meq /100 g de suelo
}

El análisis fisiográfico nos permite afirmar que los cuatro rodales se han desarrollado en terrazas bajas de la llanura aluvial, con pendientes de 20 a $35 \%$ en los rodales Caco Macaya y Agua Blanca, seguidos por pendientes de 2 a 5\%. En los rodales Agua Negra y Sábalo, las pendientes son inferiores al $2 \%$. Sin embargo, los micrositios donde se pudo observar mayor concentración de la especie en estudio, son: a orillas de las quebradas y en sitios que presentaban depresiones de nivel, favoreciendo la acumulación de agua, condiciendo con Salcedo (1986) quien menciona que la topografía es de vital importancia en la distribución de las especies (Tabla 9).

\section{Conclusiones}

a. Los cuatro rodales de Myrciaria floribunda se desarrollan en la llanura aluvial periódicamente inundable de la cuenca del río Ucayali, generalmente inundados por aguas clasificadas como "agua negra".

b. Fisiográficamente, los rodales se desarrollan en terrazas bajas, a una altitud promedio de $155 \mathrm{msnm}$.

c. La composición florística de los cuatro rodales es homegénea, con cocientes de mezcla promedio de $1 / 10$ y no superan los $30 \mathrm{~m}^{2}$ de área basal total, por lo que se consideran como sitios de baja fertilidad natural y/o rodales en etapa de sucesión temprana.

d. De acuerdo al IVIA, en los tres rodales (Caco Macaya, Agua Negra y Sábalo), Myrciaria floribunda se presenta entre las seis especies de mayor importancia ecológica, presentando una distribución natural Agrupado, con distribución horizontal no continua. e. En cuanto a la regeneración natural de Myrciaria floribunda, aún cuando no existe una escala de referencia para la especie, se considera que en promedio es alta con 3208 individuos por hectárea.

f. Los suelos de los cuatro rodales pertenecen al orden Entisols, con $\mathrm{pH}$ de 3.9 a 4.4, de textura franco arcillo limosa a arcillosa y CIC de 10 a 21 $\operatorname{cmol}(+) / k g$

Tabla 9. Clasificación fisiográfica y pendiente de los rodales.

\begin{tabular}{|c|c|c|c|c|}
\hline \multirow[t]{2}{*}{ Característica } & \multicolumn{4}{|c|}{ Rodales } \\
\hline & $\begin{array}{c}\text { Caco } \\
\text { Macaya }\end{array}$ & $\begin{array}{l}\text { Agua } \\
\text { Negra }\end{array}$ & Sábalo & $\begin{array}{c}\text { Agua } \\
\text { Blanca }\end{array}$ \\
\hline Fisiografía & $\begin{array}{c}\text { Terraza } \\
\text { baja }\end{array}$ & $\begin{array}{c}\text { Terraza } \\
\text { baja }\end{array}$ & $\begin{array}{c}\text { Terraza } \\
\text { baja }\end{array}$ & $\begin{array}{c}\text { Terraza } \\
\text { baja }\end{array}$ \\
\hline Altura (msnm) & 164 & 150 & 150 & 154 \\
\hline Pendiente & $2-35 \%$ & $0-2 \%$ & $0-2 \%$ & $2-30 \%$ \\
\hline
\end{tabular}

\section{Literatura citada}

Braun - Blanquet J. 1979. Plant sociology: The study of plant communities. Hafner, London.

Bruning E. 1970. Stand structure physiognomy and environmental factors in some lowland forest in Sarawak (Borneo). Tropical Ecology. 11 (1): 26-43.

Equihua M. 1994. Dinámica de la comunidades ecológicas. Editorial Trillas. México.

Finol H. 1971. Nuevos parámetros a considerarse en el análisis estructural de las selvas vírgenes tropicales. Revista Forestal Venezolana. 21: 29-41.

Hubbell S.P. \& Foster R.B. 1990. The fate of juvenile trees in a Neotropical forest: implications for the natural maintenance of tropical tree diversity: En: Bwa K.S. and Hadley M. (Eds), Reproductive ecology of tropical forest plants, pp.317-341, Man and the biosphere Series. vol. 7.

Iman S. 2000. Cultivo de camu camu (Myrciaria dubia H.B.K.) en la región de Loreto”. INIA - SINITTA, Lima - Perú.

Kalliola R., Puhakka M. \& Danjoy W. 1993. Amazonia Peruana. Vegetación húmeda tropical en el llano subandino Proyecto Amazonía. Univeridad de Turku. Oficina Nacional de Evaluación de recursos Naturales. Lima, Perú.

Lamprecht H. 1990. Silvicultura en los trópicos. Instituto de Silvicultura de la Universidad de Gottingen, Alemania.

López M. 2005. Exportaciones de camu camu del 2002 al 2004. Dirección General de Promoción Agraria Ministerio de Agricultura, Lima.

Malleux J. 1971. Estratificación forestal con uso de fotografías aéreas. Universidad Nacional Agraria, Lima, Perú. V.1.

Malleux J. 1982. Inventarios forestales en bosques tropicales. Universidad Nacional Agraria. Lima, Perú.

Mendoza O., Picon C. \& Vásquez C. 1989. Informe de la expedición de recolección de germoplasma de Camu 
camu (Myrciaria dubia) en la Amazonía Peruana. Informe técnico $\mathrm{N}^{\circ} 11$, Programa de Investigación en Cultivos Tropicales, INIAA Lima.

Pinedo M.R., Riva C., Delgado A. \& López J. 2001. "Sistema de producción de camu camu en restinga" Manual técnico. Instituto de Investigaciones de la Amazonia Peruana IIAP Iquitos.

Sabogal C. 1980. Estudio de caracterización ecológico silvicultural del Bosque "Copal" - Jenaro Herrera (Loreto - Perú). Tesis para optar el título de Ing. Forestal UNALM - Lima.

Salcedo G. 1986. Estudio ecológico y estructural del bosque "Los Espaveles", Turrialba, Costa Rica. Tesis Mg. Sc.
Programa Posgrado de la Universidad de Costa Rica. CATIE.

Universidad Nacional de Ucayali. 2002. Datos meteorológicos de la Estación Principal de Pucallpa. Calleria, Coronel Portillo.

Villachica H. 1996. El cultivo de Camu camu (Myrciaria dubia) H.B.K. Mc Vaugh en la Amazonía Peruana. Tratado de Cooperación Amazónica.

Apéndice de figuras y tablas citadas en el texto.
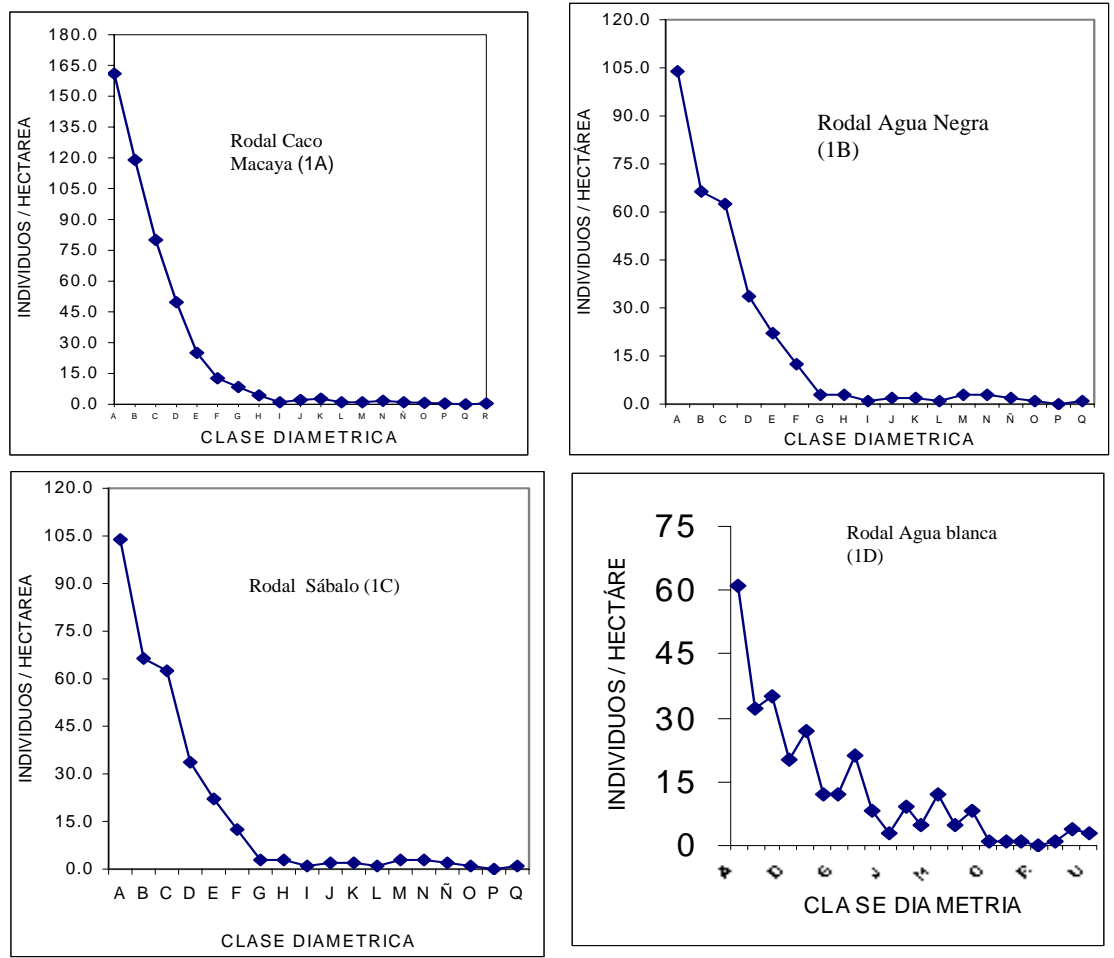

Figura 1. Distribución diamétrica de los cuatro rodales naturales de Myrciaria floribunda. 

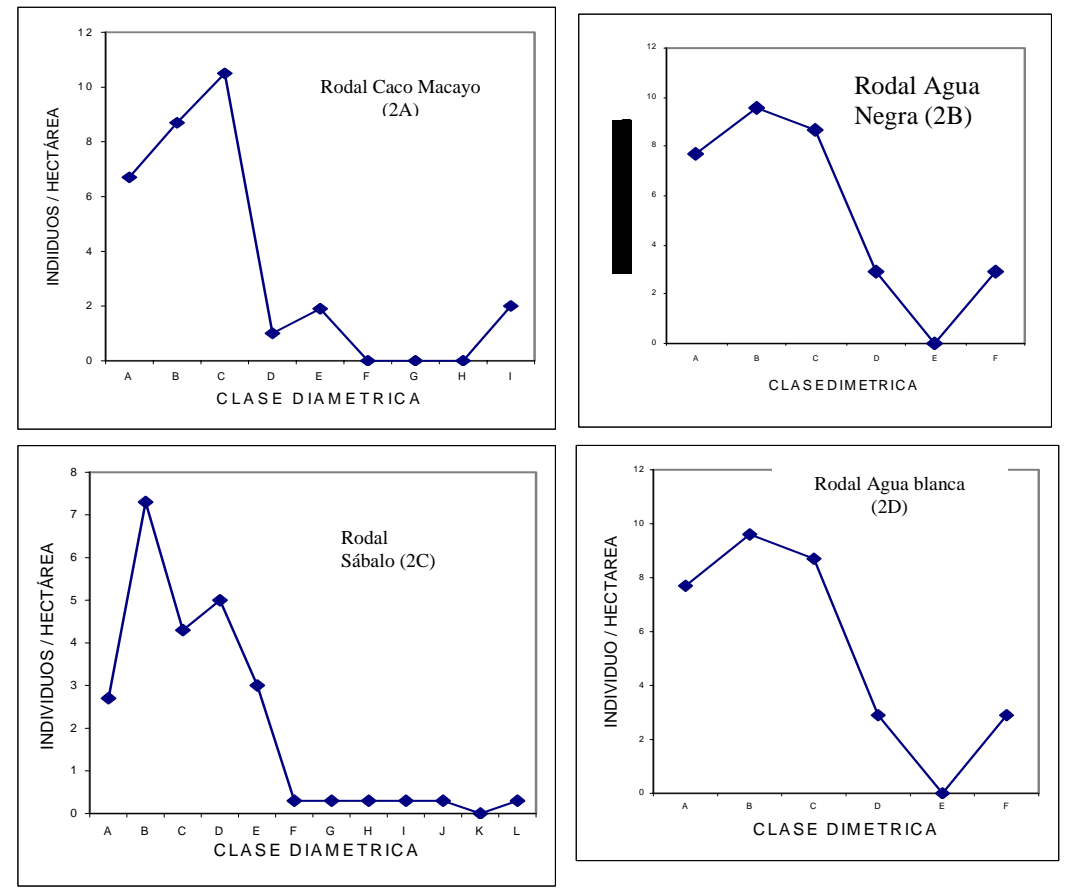

Figura 2. Distribución diamétrica de Myrciaria floribunda en los cuatro rodales naturales.

Tabla 6. Índice de valor de importancia de los cuatro rodales

\begin{tabular}{|c|c|c|c|}
\hline & Especie & IVIA & $\%$ \\
\hline 1 & Abuta grandifolia & 101.3 & 20.26 \\
\hline 2 & ND 10 & 75.93 & 14.98 \\
\hline 3 & Rollina cuspidate $M$ & 44.01 & 8.802 \\
\hline 4 & ND 10 & 10.14 & 8.028 \\
\hline 5 & Pitadenia sp & 34.41 & 6.882 \\
\hline 6 & Myrciaria floribunda & 32.94 & 6.588 \\
\hline 7 & Otras especies & 172.27 & 34.54 \\
\hline \multicolumn{4}{|c|}{ 2. Rodal Agua Negra } \\
\hline & Especie & IVIA & $\%$ \\
\hline 1 & Ficus $s p$ & 91.96 & 18.39 \\
\hline 2 & Myrciaria floribunda & 67.47 & 13.49 \\
\hline 3 & Abuta grandifolia & 35.06 & 7.01 \\
\hline 4 & Cecropia sp & 34.09 & 6.81 \\
\hline 5 & Couratari $s p$ & 25.25 & 5.05 \\
\hline 6 & Inga sp & 16.92 & 3.38 \\
\hline 7 & Otras especies & 229.25 & 45.85 \\
\hline
\end{tabular}

\begin{tabular}{|c|c|c|c|}
\hline & Especie & IVIA & $\%$ \\
\hline 1 & ND 3 & 66.6 & 13.32 \\
\hline 2 & Abuta grandifolia & 52.7 & 10.54 \\
\hline 3 & Myrciaria floribunda & 52.1 & 10.42 \\
\hline 4 & Piptadenia sp & 50 & 10 \\
\hline 5 & Ficus $s p$ & 7.62 & 7.62 \\
\hline 6 & Inga $s p$ & 7.6 & 7.6 \\
\hline 7 & Otras especies & 40.5 & 10.5 \\
\hline \multicolumn{4}{|c|}{ 4. Rodal Agua blanca } \\
\hline & Especie & IVIA & $\%$ \\
\hline 1 & Crataeva tapia & 48.7 & 9.74 \\
\hline 2 & Tripalris longifolia & 46.9 & 9.38 \\
\hline 3 & ND 10 & 36.8 & 7.36 \\
\hline 4 & Abuta grandifolia & 36.7 & 7.34 \\
\hline 5 & Rollina cuspidate $M$ & 34.9 & 6.98 \\
\hline 6 & ND 8 & 34.4 & 6.88 \\
\hline 7 & Otras especies & 261.6 & 52.32 \\
\hline
\end{tabular}

\footnotetext{
${ }^{1}$ Investigador Programa de Ecosistemas Terrestres IIAP, jlopez@iiap.org.pe

${ }^{2}$ Tesista Facultad Ciencias Forestales UNU, ebicerra99@hotmail.com

${ }^{3}$ Profesor Principal Facultad Ciencias Forestales UNU, ediaz@unu.edu.pe
} 